\title{
Deep anterior lamellar keratoplasty with manual small-incision cataract surgery: a modification of the triple procedure
}

\author{
Pawan Prasher \\ Department of Ophthalmology, Sri Guru Ram Das Institute of Medical Sciences \\ and Research, Vallah, Amritsar, Punjab, India
}

\begin{abstract}
We describe a modified surgical technique for performing the triple procedure that combines deep anterior lamellar keratoplasty with manual small-incision cataract surgery. This technique offers advantages in terms of undergoing a single surgery as compared to the staged procedure, preservation of the host Descemet's membrane and endothelium, and ability to perform cataract surgery in a closed-chamber setting.
\end{abstract}

Keywords: deep anterior lamellar keratoplasty, manual small-incision cataract surgery, triple procedure

Penetrating keratoplasty (PKP) triple is a well-established surgical procedure used for the management of patients with comorbidities of corneal opacities and cataract. ${ }^{1}$ It involves making a full-thickness circular incision incorporating the pathology in the host cornea, performing cataract extraction and intraocular lens (IOL) implantation by open-sky technique, followed by suturing the donor cornea to the host bed. Over the last two decades, there has been a paradigm shift from full-thickness PKP to lamellar keratoplasty, which is aimed at replacing only the diseased layers of the cornea while preserving the normal ones. This has posed new challenges in terms of offering combined procedures to the patients with comorbidities. Although a combination of deep anterior lamellar keratoplasty (DALK) with phacoemulsification has been described by various surgeons, ${ }^{2-5}$ phacoemulsification may not always be possible for logistic reasons, or it may be difficult due to patient-related factors such as small pupil, hard cataract, or weak zonules. Manual small-incision cataract surgery (MSICS) technique is a viable alternative to phacoemulsification and is routinely used to perform cataract surgery in developing countries like India. Herein, we describe a modification of the triple procedure that combines DALK with MSICS and offers advantages of both surgeries while obviating the need for multiple surgeries in selected patients with comorbidities.

Correspondence: Dr. Pawan Prasher, Amritsar Eye Hospital, GNDU Shopping Complex, GT Road, Amritsar, Punjab 143001, India.

E-mail:pawanprasher@yahoo.com 


\section{Case report}

A 65-year-old male, farmer by occupation, presented with bilateral corneal opacities, attributed to corneal scarring and degeneration secondary to old trachomatous infection, and a cataract in the right eye. The opacity appeared to be predominant in the anterior cornea with relative sparing of posterior stroma and Descemet membrane. The left eye had undergone cataract surgery 2 years back; however, the patient was not satisfied with the poor visual outcome which was attributed to visually significant corneal opacity in that eye. Best spectacle-corrected visual acuity (BSCVA) was 6/60 in the right eye and 6/24 in the left eye. The intraocular pressure was 14 and $16 \mathrm{mmHg}$ in the right and left eye. The remaining ocular and systemic examination was unremarkable. As the opacity was denser in the right eye, a combined triple procedure was planned after getting informed consent from the patient. The IOL power was measured using a presumed keratometry reading of $44 \mathrm{D}$ as is routinely done at our institute for the triple procedures.

\section{Surgical technique}

The patient was taken up for surgery after a donor corneal button was procured from one of the approved eye banks under Central Distribution System of India. Under peribulbar anesthesia, a superior rectus bridle suture was placed and a fornix-based conjunctival flap was raised along the superior limbus. A 4-mm-long, partial thickness, scleral incision was made $2 \mathrm{~mm}$ posterior to the superior limbus. A corneoscleral tunnel was constructed up to the limbus with a crescent blade. An oblique side-port incision was made inferotemporally and a large air bubble was injected into anterior chamber to make eye firm. The stromal dissection was carried out all around the cornea up to 1.5 to $2 \mathrm{~mm}$ inside the limbus with a Morlet dissector (Duckworth and Kent, Hertfordshire, England), using a technique described by Melles. ${ }^{6}$ This technique makes use of the specular reflection to achieve dissection as close to Descemet membrane as possible (Fig. 1). The scleral incision was subsequently closed with three interrupted 10-0 nylon sutures. Most of the air bubble was subsequently aspirated leaving behind a small bubble in the anterior chamber to aid in the visualization of the integrity of Descemet membrane. A viscoelastic was injected into the scleral tunnel to separate the anterior and posterior corneal lamellae. The movement of air bubble to the periphery of anterior chamber confirmed the separation and posterior distension of the posterior lamella. Anterior corneal button was removed using an 8-0 trephine and corneal scissors, as is routinely done for conventional PKP. The surgeon now moved to the temporal side of the patient and a fornix-based conjunctival flap was fashioned temporally. A temporal sclerocorneal tunnel was constructed as is routinely done in MSICS up to $1 \mathrm{~mm}$ inside the temporal limbus 

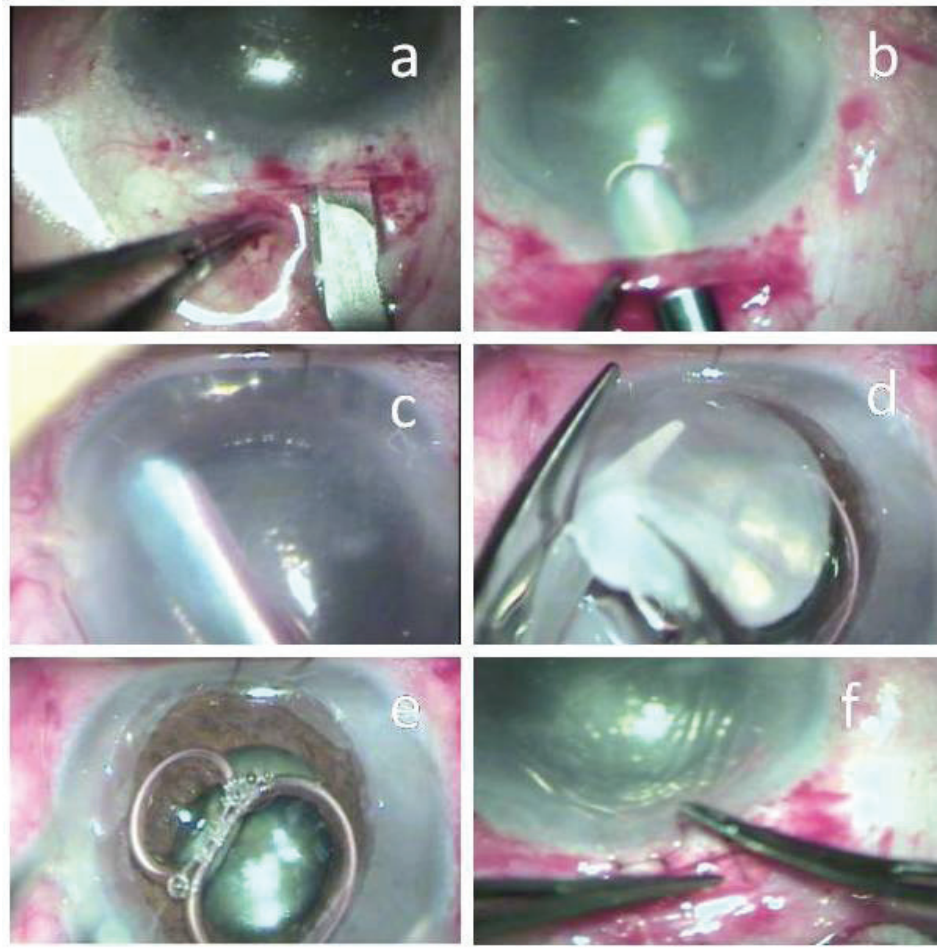

Fig. 1. Intraoperative pictures describing various steps of lamellar dissection:

(a) sclerocorneal tunnel construction. (b and c) Stromal dissection.

(d) Excision of anterior corneal lamella. (e) Air bubble in anterior chamber suggestive of preservation of integrity of Descemet membrane.

(f) Suturing of the scleral incision.

taking care not to extend the tunnel to the plane of corneal dissection. Subsequent maneuvers including hydrodissection, nucleus delivery using viscoexpression, cortical aspiration, and implantation of single-piece polymethyl methacrylate IOL were performed gently in an attempt to prevent any abrupt changes in the anterior chamber pressure (Fig. 2). The residual stroma offered excellent view for performing various steps. During the cortical aspiration, a note was made of posterior movement of posterior lamella towards the aspiration tip which was easily prevented by manual reduction of the speed of aspiration. The temporal sclera incision was closed with a 10-0 nylon suture to avoid any potential wound gape by traction from subsequent corneal sutures to be used to secure donor cornea to the host bed. The anterior stromal bed was meticulously washed to remove any viscoelastic material or debris. The donor cornea preparation 

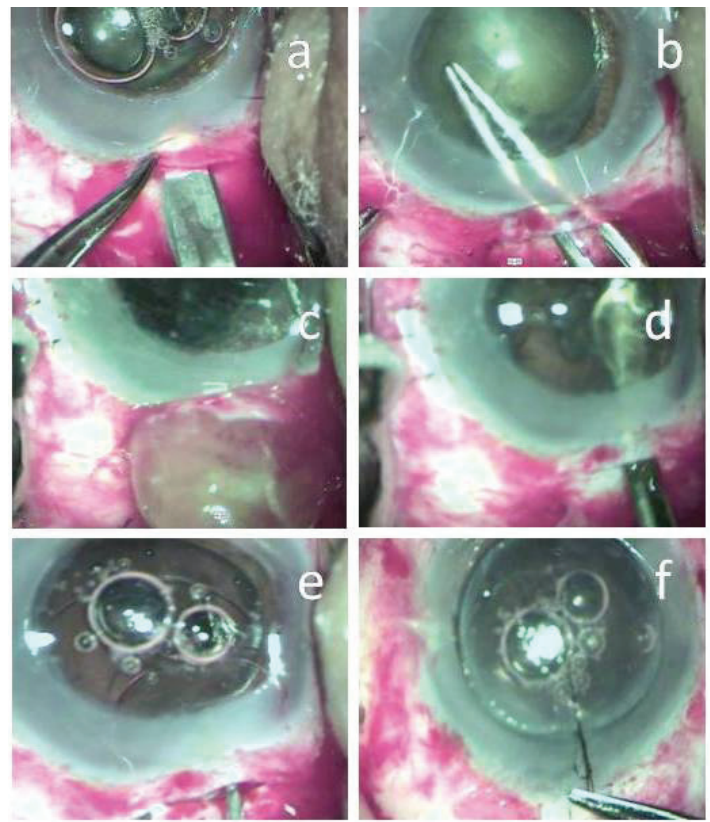

Fig. 2. Intraoperative pictures describing various steps of cataract surgery and donor placement: (a) temporal sclerocorneal tunnel construction. (b) Capsulorrhexis. (c) Nucleus delivery. (d) Cortex aspiration. (e) IOL implantation. (f) Suturing of donor cornea to the host bed.

included complete stripping of Descemet's membrane and endothelium using dry Weck-Cels. An 8.25-mm trephine was subsequently used to prepare the donor corneal button which was sutured to the host bed using a combination of 12 interrupted and a 12-bite single running suture. The conjunctival flaps were closed and subconjunctival gentamicin and dexamethasone combination was injected before closing the eye.

\section{Postoperative course}

The early postoperative treatment included topical moxifloxacin four times daily, topical prednisolone eye drops six times daily and topical lubricants six times daily for 3 weeks. Moxifloxacin was subsequently discontinued and prednisolone drops were gradually tapered to twice daily over a period of 6 weeks. The early postoperative period was significant for a persistent epithelial defect which was treated with a bandage contact lens application. He had a stromal rejection episode at 10 months which was attributed to a loose suture and was controlled 

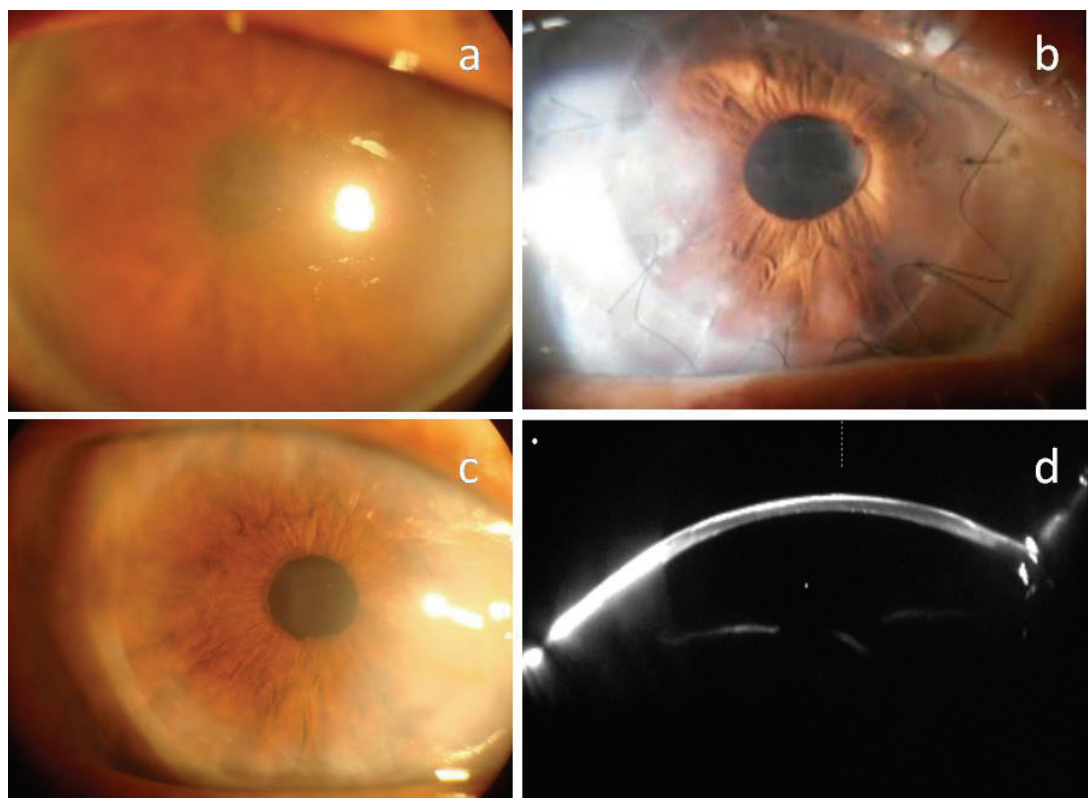

Fig. 3. (a) Slit-lamp pictures of the cornea show dense corneal opacity preoperatively.

(b) At 3 months, graft is clear with mild interface haze. (c) At last follow-up more than 3 years after surgery, graft has remained clear with mild persistent interface haze. (d) Scheimpflug imaging shows minimal reflectivity at the interface.

by intensive topical and oral steroids along with suture removal. At his last visit 3.5 years post-operatively BSCVA was $6 / 12$ with a manifest refraction of -0.75 $D-2 D \times 170$. The graft looked healthy apart from mild interface haze, which was confirmed on Scheimpflug imaging, and the IOL was well-centered (Fig. 3). The specular microscopy was not available in the current case.

\section{Discussion}

We describe a modification of the triple procedure that combines DALK with MSICS. It offers advantages in terms of early visual rehabilitation by treating corneal disease and cataract in the same sitting as compared to the staged procedure. The advantages of DALK over PKP include preservation of host endothelium, which carries lower risk of endothelial rejection and hence lesser need for long-term steroids, leading to lower incidence of postoperative glaucoma, cataract and secondary infection, and better wound integrity in situations of inadvertent trauma. ${ }^{1-10}$ The drawbacks of the current technique include interface haze as a result of scarring due to residual stroma as Descemet's membrane is not bared, unlike the more preferred Anwar's big-bubble technique in which 
Descemet membrane is bared.7 Although previous authors have successfully performed phacoemulsification with DALK using big-bubble technique, there has been a concern regarding the unpredictability of the type of bubble formation. While it was possible to perform phacoemulsification when big-bubble formation occurred at the pre-Descemet level; the residual tissue in a big-bubble formed at the level of Descemet membrane could not withstand the raised introaocular pressure during cataract surgery, leading to its rupture. ${ }^{4}$ In such a situation, having some residual stromal tissues may offer advantage in providing sufficient strength to the posterior corneal lamella for performing combined cataract surgery in a closed chamber. Although interface haze has been a concern with manual dissection, a randomized controlled trial between Melles' versus Anwar technique did not show any significant difference in terms of visual acuity and refractive outcomes between the two techniques, although contrast sensitivity was better with Anwar technique. ${ }^{8}$ Another study, however, found a correlation between reduced visual acuity and residual stromal thickness, wherein they reported that more than $80 \mu \mathrm{m}$ of residual stroma is associated with significantly reduced visual acuity. ${ }^{9}$

The current technique makes use of a sclerocorneal tunnel to perform the cataract surgery in a closed chamber as compared to open-sky method in routine triple procedure. In a closed-chamber setting, the surgeon has a greater control in performing various steps like capsulorrhexis, nucleus delivery, cortex aspiration, and IOL placement in the capsular bag, and there is a less risk of potentially devastating complications associated with open-sky method like expulsive hemorrhage and choroidal effusion. ${ }^{11}$ Although phacoemulsification is considered a gold standard for cataract surgery, efficacy and safety of MSICS has been well established, especially in developing countries. ${ }^{12-14}$ Although phacoemulsification can also be done with DALK, we believe MSICS is a viable alternative in situations where phacoemulsification may be difficult or risky like in eyes with hard cataracts, small pupils, and weak zonules, or where phacoemulsification may not be possible or available for logistic reasons. MSICS has the advantage of being a manual procedure devoid of complications related to ultrasound energy and is relatively inexpensive. In the current technique, cataract surgery component can be added to DALK with minimal cost in terms of additional equipments or instruments. Although phacoemulsification offers advantage of inducing less astigmatism, it may not always transform into functional improvement for the patients. ${ }^{14}$ Also, in a combined triple procedure, the final astigmatism is more likely to be determined by donor graft-host interaction rather than by the cataract surgery incision alone. The current technique would be suitable for a subset of patients with coexisting anterior corneal pathologies not involving the Descemet's membrane and visually significant cataracts in situations where 
phacoemulsification machine and foldable IOLs may not be available due to logistics or cost factors.

In conclusion, despite some of its inherent drawbacks, combined DALK with MSICS technique may be useful in select patients with comorbidities and provides a viable alternative to the corneal surgeons planning to perform triple procedure in such patients.

\section{References}

1. Oie Y, Nishida K. Triple procedure: cataract extraction, intraocular lens implantation, and corneal graft. Curr Opin Ophthalmol. 2017;28:63-66.

2. Muraine MC, Collet A, Brasseur G. Deep lamellar keratoplasty combined with cataract surgery. Arch Ophthalmol. 2002;120:812-815.

3. Senoo T. Combined surgery with deep lamellar keratoplasty. Semin Ophthalmol. 2001;16:126-136.

4. Zaki AA, Elalfy MS, Said DG, Dua HS. Deep anterior lamellar keratoplasty—triple procedure: a useful clinical application of the pre-Descemet's layer (Dua's layer). Eye (Lond). 2015;29:323-326.

5. Panda A, Sethi HS, Jain M, Nindra Krishna S, Gupta AK. Deep anterior lamellar keratoplasty with phacoemulsification. J Cataract Refract Surg. 2011;37:122-126.

6. Melles GR, Lander F, Rietveld FJ, Remeijer L, Beekhuis WH, Binder PS. A new surgical technique for deep stromal, anterior lamellar keratoplasty. Br J Ophthalmol. 1999;83:327-333.

7. Anwar M, Teichmann KD. Big-bubble technique to bare Descemet's membrane in anterior lamellar keratoplasty. J Cataract Refract Surg. 2002;28:398-403.

8. Baradaran-Rafii A, Eslani M, Sadoughi MM, Esfandiari H, Karimian F. Anwar versus Melles deep anterior lamellar keratoplasty for keratoconus: a prospective randomized clinical trial. Ophthalmology. 2013;120:252-259.

9. Ardjomand N, Hau S, McAlister JC, et al. Quality of vision and graft thickness in deep anterior lamellar and penetrating corneal allografts. Am J Ophthalmol. 2007;143:228-235.

10. Prasher $P$, Muftuoglu $O$, Mootha VV. Traumatic graft dehiscence after anterior lamellar keratoplasty. Cornea. 2009;28:240-242.

11. Chen W, Ren Y, Zheng Q, Jhanji V. Stabilized triple procedure for management of coexisting corneal opacity and cataract. J Cataract Refract Surg. 2014;40:1966-1970.

12. Gogate PM, Kulkarni SR, Krishnaiah S, et al. Safety and efficacy of phacoemulsification compared with manual small-incision cataract surgery by a randomized controlled clinical trial: six-week results. Ophthalmology. 2005;112:869-874.

13. Ruit $S$, Tabin G, Chang D, et al. A prospective randomized clinical trial of phacoemulsification vs manual sutureless small-incision extracapsular cataract surgery in Nepal. Am J Ophthalmol. 2007;143:32-38.

14. Gogate P, Optom JB, Deshpande S, Naidoo K. Meta-analysis to compare the safety and efficacy of manual small incision cataract surgery and phacoemulsification. Middle East Afr J Ophthalmol. 2015;22:362-369. 